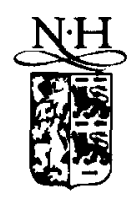

ELSEVIER

Comput. Methods Appl. Mech. Engrg. 143 (1997) 49-67

\title{
A finite element formulation for incompressible flow problems using a generalized streamline operator
}

\author{
Marcela A. Cruchaga*, Eugenio Oñate \\ International Center for Numerical Methods in Engineering, E.T.S. d'Enginyers de Camins, Canals $i$ Ports,
}

Received 23 May 1995; revised 21 February 1996

\begin{abstract}
A finite element formulation for solving incompressible flow problems is presented. In this paper, the generalized streamline operator presented by Hughes et al. (Comput. Methods Appl. Mech. Engrg. (1986) 58 305-328) for compressible flows is adapted to the incompressible Navier-Stokes equations. This new methodology allows the use of equal order interpolation for the unknowns of the problem: velocity and pressure. In this context, the definition of the 'upwinding tensor' does not require parametcrs defined outside this model. This formulation has been checked in classical tests with satisfactory results. Finally, a moving surface problem (Cruchaga et al., Comput. Numer. Methods Engrg. (1986) 59: 85-99) is also presented.
\end{abstract}

\section{Introduction}

In the present work a numerical formulation able to deal with incompressible flow problems is developed. The difficulties in the numerical solution of the Navier-Stokes equations are well known: uscillations appear in the results when the convective term becomes relevant and mathematical requirements impose restrictions on the choice of the discrete approximation functions although, however, a recent formulations allow to overcome such restrictions [1-4]. In the context of the finite element method, a Galerkin Least Squares type formulation using a generalized streamline operator [5] applied to the incompressible flow case is presented. This technique enables the use of equal interpolation function for the primitive variables of the problem: velocity and pressure. In this case, the standard penalization methods necessary to fulfil the incompressibility equation are not required.

The choice of the upwinding parameters, crucial to obtain stable and convergent formulations, involves several works and discussions [4,6-9]. In this paper, a new design of these parameters is obtained in the framework of the generalized streamline operator (GSO) presented by Hughes et al. [5] extending the methodology initially developed for compressible flows [9]. The GSO consists, basically, in writing the Navier-Stokes equations in the advective eigenvector system. In this basis, the diffusivity matrix is lumped leading to an uncoupled governing equations (considering a constant viscosity) and, therefore, the computation of the upwinding coefficient at each direction can be performed in the standard manner. Further, a diagonal upwinding tensor is obtained and, transforming this tensor back to the original system, the weighting perturbation function can be defined.

The governing equations for the incompressible flow problem and the corresponding weak form are

\footnotetext{
* Corresponding author.
} 
described in Section 2. In Section 3, the methodology followed to compute the upwinding tensor is presented. It is important to note that this tensor does not require tuning parameters defined outside this model. Further, the resulting finite element formulation is briefly described in Section 4 .

A brief description of the numerical strategy is performed in Section 5 where an incremental-iterative solution strategy has been implemented such that the convergence criterion is written in terms of the norm of the residual vèctor.

In Section 6, the driven cavity flow problem is analysed at different Reynolds' numbers and a comparative analysis with other techniques is performed. The backward-facing step flow is also studied in order to compare with experimental results. Finally, a two-liquid interface problem is solved using the presented methodology.

\section{Governing equations and weak form}

The basic formulation for incompressible flow problems considering a Newtonian fluid are described by the Cauchy's equation of motion and the continuity equation which are written in Cartesian components as

$$
\rho \frac{\partial u_{i}}{\partial t}+\rho \frac{\partial u_{i}}{\partial x_{j}} u_{j}+\frac{\partial p}{\partial x_{i}}-\frac{\partial\left(2 \mu \varepsilon_{i j}\right)}{\partial x_{j}}=\rho b_{i} \quad \text { in } \Omega \times[0, T]
$$

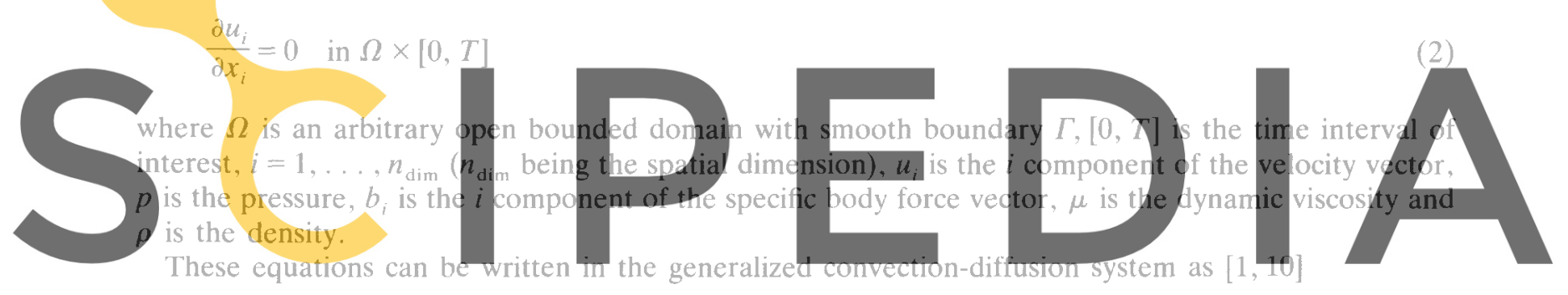

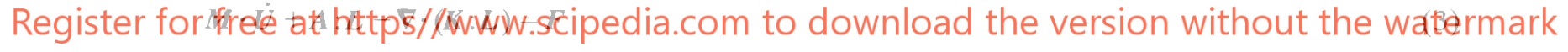

and in indicial notation as

$$
M_{i m} \dot{u}_{m}+A_{i m n} L_{m n}-\nabla_{j} K_{j i m n} L_{m n}=F_{i}
$$

where

- $U$ is the unknown vector, $\boldsymbol{U}=\left[u_{1}, u_{2}, u_{3}, u_{4}\right]$ with $u_{4}=p$. Now, $i=1, \ldots, n_{\mathrm{dim}}+1, m=$ $1, \ldots, n_{\mathrm{dim}}+1, n=1, \ldots, n_{\mathrm{dim}}$ and $j=1, \ldots, n_{\mathrm{dim}}$.

- $\boldsymbol{A}=A_{i m n}$ is the generalized advection tensor. For a fixed value of $n=\bar{n}, \boldsymbol{A}_{\bar{n}}=\left[A_{i m \bar{n}}\right]$ is defined as

$$
\boldsymbol{A}_{1}=\left[\begin{array}{llll}
\rho u_{1} & 0 & 0 & 1 \\
0 & \rho u_{1} & 0 & 0 \\
0 & 0 & \rho u_{1} & 0 \\
1 & 0 & 0 & 0
\end{array}\right] \quad \boldsymbol{A}_{2}=\left[\begin{array}{llll}
\rho u_{2} & 0 & 0 & 0 \\
0 & \rho u_{2} & 0 & 1 \\
0 & 0 & \rho u_{2} & 0 \\
0 & 1 & 0 & 0
\end{array}\right] \quad \boldsymbol{A}_{3}=\left[\begin{array}{llll}
\rho u_{3} & 0 & 0 & 0 \\
0 & \rho u_{3} & 0 & 0 \\
0 & 0 & \rho u_{3} & 1 \\
0 & 0 & 1 & 0
\end{array}\right]
$$

- $\boldsymbol{K}=K_{\text {jimn }}$ is a generalized diffusion tensor. For a fixed value of $n=\bar{n}$ and $j=\bar{j}, \boldsymbol{K}_{\bar{j} \bar{n}}=\left[K_{\overline{j i m} \bar{n}}\right]$ is defined as

$$
\boldsymbol{K}_{11}=\left[\begin{array}{llll}
2 \mu & 0 & 0 & 0 \\
0 & \mu & 0 & 0 \\
0 & 0 & \mu & 0 \\
0 & 0 & 0 & 0
\end{array}\right] \quad \boldsymbol{K}_{12}=\left[\begin{array}{llll}
0 & \mu & 0 & 0 \\
0 & 0 & 0 & 0 \\
0 & 0 & 0 & 0 \\
0 & 0 & 0 & 0
\end{array}\right] \quad \boldsymbol{K}_{13}=\left[\begin{array}{llll}
0 & 0 & \mu & 0 \\
0 & 0 & 0 & 0 \\
0 & 0 & 0 & 0 \\
0 & 0 & 0 & 0
\end{array}\right]
$$




$$
\begin{aligned}
\boldsymbol{K}_{21}=\left[\begin{array}{llll}
0 & 0 & 0 & 0 \\
\mu & 0 & 0 & 0 \\
0 & 0 & 0 & 0 \\
0 & 0 & 0 & 0
\end{array}\right] & \boldsymbol{K}_{22}=\left[\begin{array}{llll}
\mu & 0 & 0 & 0 \\
0 & 2 \mu & 0 & 0 \\
0 & 0 & \mu & 0 \\
0 & 0 & 0 & 0
\end{array}\right] & \boldsymbol{K}_{23}=\left[\begin{array}{llll}
0 & 0 & 0 & 0 \\
0 & 0 & \mu & 0 \\
0 & 0 & 0 & 0 \\
0 & 0 & 0 & 0
\end{array}\right] \\
\boldsymbol{K}_{31}=\left[\begin{array}{llll}
0 & 0 & 0 & 0 \\
0 & 0 & 0 & 0 \\
\mu & 0 & 0 & 0 \\
0 & 0 & 0 & 0
\end{array}\right] & \boldsymbol{K}_{32}=\left[\begin{array}{llll}
0 & 0 & 0 & 0 \\
0 & 0 & 0 & 0 \\
0 & \mu & 0 & 0 \\
0 & 0 & 0 & 0
\end{array}\right] & \boldsymbol{K}_{33}=\left[\begin{array}{llll}
\mu & 0 & 0 & 0 \\
0 & \mu & 0 & 0 \\
0 & 0 & 2 \mu & 0 \\
0 & 0 & 0 & 0
\end{array}\right]
\end{aligned}
$$

- $L$ is the spatial gradient tensor of the unknown defined as $L_{m n}=\partial u_{m} / \partial x_{n}$.

- $\nabla$ is the gradient operator $\nabla_{j}(\cdot)=\partial(\cdot) / \partial x_{j}$.

- $\boldsymbol{M}$ is the generalized mass tensor:

$$
M=\left[\begin{array}{llll}
\boldsymbol{\rho} & 0 & 0 & 0 \\
0 & \rho & 0 & 0 \\
0 & 0 & \rho & 0 \\
0 & 0 & 0 & 0
\end{array}\right]
$$

\section{- $F$ is the body force vector.}

$$
F=\left[\rho b_{1}, \rho b_{2}, \rho b_{3}, 0\right]
$$

The formal problem consists of finding $U$ verifying the system (3) such that
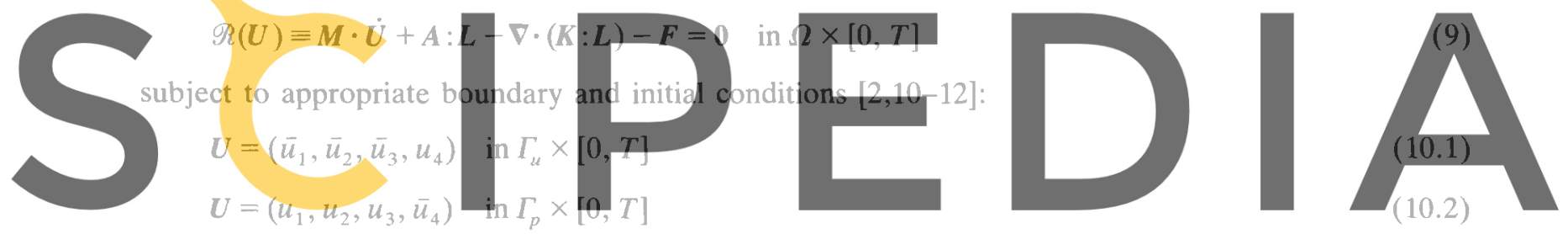

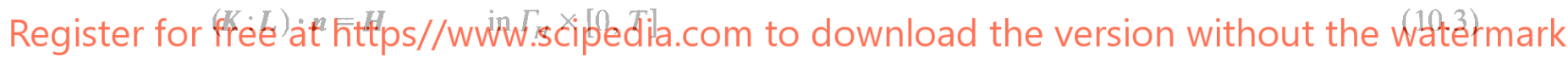 $U_{0}=U(x, 0) \quad$ in $\Omega$

where $\boldsymbol{H}$ is the prescribed deviatoric stress tensor, $\boldsymbol{n}$ is the outward unit vector normal to $\Gamma_{H}$. Besides, $\Gamma_{u}, \Gamma_{p}, \Gamma_{H}$ are the parts of $\Gamma$ on which the velocity, the pressure and the deviatoric part of the stress tensor are prescribed, respectively $\left(\Gamma_{u} \cup \Gamma_{H}=\Gamma\right.$ and $\left.\Gamma_{u} \cap \Gamma_{H}=\emptyset\right)$; see Fig. 1.

In order to obtain the weak form of this initial boundary value problem, the perturbation function added to the standard Galerkin weighting function is defined as [5]

$$
\boldsymbol{P}(\boldsymbol{\Psi})=\boldsymbol{\tau} \cdot \mathscr{R}(\boldsymbol{\Psi})
$$

where $\mathscr{R}$ defined in Eq. (9) is applied to the test function $\boldsymbol{\Psi}$ and $\tau$ is the second-order 'upwinding

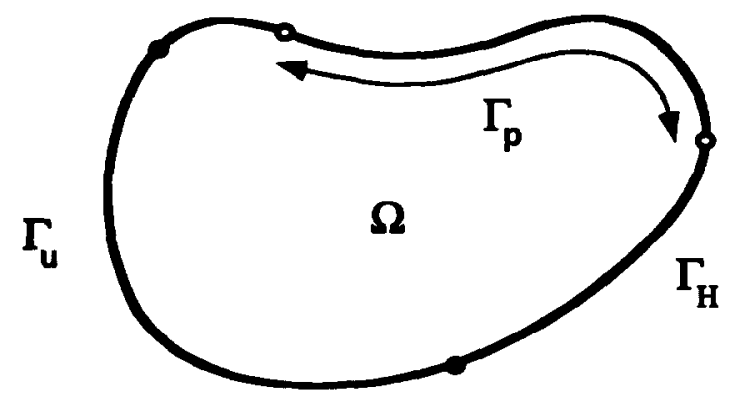

Fig. 1. Arbitrary domain and its boundary. 
tensor' that will be defined in Section $3\left(\tau=\tau_{i q}\right.$, with $i=1, \ldots, n_{\mathrm{dim}}+1$ and $\left.q=1, \ldots, n_{\mathrm{dim}}+1\right)$. In the present work, only the convective part of the perturbation function is used. Therefore, the weighting function is time-independent, the effect of the diffusive term involving second derivatives of the unknowns is neglected, and the source term does not depend on the variables of the problem. Accordingly, the perturbation function is written as $[5,10]$

$$
\boldsymbol{P}(\boldsymbol{\Psi})=\boldsymbol{\tau} \cdot(\boldsymbol{A}: \boldsymbol{L}(\boldsymbol{\Psi}))=\tau_{i q} A_{q m n} L_{m n}(\boldsymbol{\Psi})=\beta_{i m n} L_{m n}(\boldsymbol{\Psi})=\beta: L(\boldsymbol{\Psi})
$$

Finally, the variational form of the problem defined by Eqs. (9) and (10) is assumed to be [5]

$$
\begin{aligned}
& \int_{\Omega} \boldsymbol{\Psi} \cdot \mathscr{R}(U) \mathrm{d} \Omega+\sum_{e} \int_{\Omega_{e}} \boldsymbol{P}(\boldsymbol{\Psi}) \cdot \mathscr{R}(U) \mathrm{d} \Omega_{e} \\
& \quad+\int_{\Gamma_{H}} \boldsymbol{\Psi} \cdot[(K: L(U)) \cdot \boldsymbol{n}-H] \mathrm{d} \Gamma+\int_{r} \boldsymbol{\Psi} \cdot\left(U-U^{*}\right) \mathrm{d} \Gamma=0
\end{aligned}
$$

\section{Upwinding tensor computation}

In order to obtain the "upwinding tensor" $\tau$, the following advection-diffusion system is considered

$$
\boldsymbol{A}: \boldsymbol{L}-\boldsymbol{\nabla} \cdot(\boldsymbol{K}: \boldsymbol{L})=\mathbf{0}
$$

that is, in fact, system (3) neglecting the source term and assuming steady-state conditions.

Due to the geometric mapping from the parent domain to the elemental domain related to the isoparametric finite elements, Eq. (14) can be written in this local elemental system $\xi$. Taking this fact

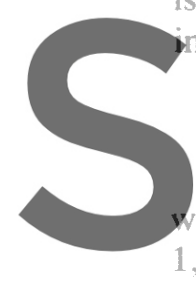

ynto account, the following

With these considerations, the generalized convective term can be written as

Register for free at https//www.scipedia.com to download the version without the watermark
\[ A: L(U)=A_{i m n} L_{m n}=A_{i m n} L_{m r} J_{r n}=A: L(U) \]

where $\hat{A}$ is defined by

$$
\hat{\boldsymbol{A}}=\hat{A}_{i m r}=A_{i m n} J_{r n}=\boldsymbol{A} \cdot \boldsymbol{J}^{\mathrm{T}}
$$

that, for a fixed value $r=\bar{r}, \hat{A}_{\bar{r}}$ is

$$
\hat{\boldsymbol{A}}_{\bar{r}}=\left[\hat{A}_{i m \bar{r}}\right]=\left[\begin{array}{llll}
\hat{a}_{\bar{r}} & 0 & 0 & J_{\bar{r} 1} \\
0 & \hat{a}_{\bar{r}} & 0 & J_{\bar{r} 2} \\
0 & 0 & \hat{a}_{\bar{r}} & J_{\bar{r} 3} \\
J_{\bar{r} 1} & J_{\bar{r} 2} & J_{\bar{r} 3} & 0
\end{array}\right]
$$

where $\hat{a}_{\dot{r}}=\rho L_{\bar{j}} u_{j}$.

In this context, the generalized diffusion term is

$$
\boldsymbol{\nabla} \cdot \boldsymbol{K}: \boldsymbol{L}=\nabla_{j} K_{j i m n} L_{m n}=\hat{\nabla}, J_{r j} K_{j i m n} \hat{L}_{m s} J_{s n}=\hat{\nabla}_{r} \hat{K}_{r i m s} \hat{L}_{m s}=\hat{\boldsymbol{\nabla}} \cdot \hat{\boldsymbol{K}}: \hat{\boldsymbol{L}}
$$

where $s=1, \ldots, n_{\mathrm{dim}}, \hat{\nabla}_{r}(\cdot)=\partial(\cdot) / \partial \xi_{r}$, and

$$
\hat{\boldsymbol{K}}=\hat{K}_{r i m s}=J_{r j} K_{j i m n} J_{s n}=\boldsymbol{J} \cdot \boldsymbol{K} \cdot \boldsymbol{J}^{\mathrm{T}}
$$

As mentioned before, Eq. (14) is written in the local system $\xi$ as

$$
\hat{\boldsymbol{A}}: \hat{\boldsymbol{L}}-\hat{\boldsymbol{\nabla}} \cdot \hat{\boldsymbol{K}}: \hat{\boldsymbol{L}}=\mathbf{0}
$$


Moreover, the matrix-valued $p$-norm of $\hat{\boldsymbol{A}}$ is defined as [5]

$$
|\hat{\boldsymbol{A}}|_{p}=\left\{\sum_{r}\left|\hat{\boldsymbol{A}}_{r}\right|^{p}\right\}^{1 / p}
$$

such that $p$ can be chosen in the integer interval $[1, \infty]$, and $\left|\hat{A}_{r}\right|^{p}$ are obtained solving the $\hat{\boldsymbol{A}}_{r}$ eigenproblem as

$$
\left|\hat{\boldsymbol{A}_{r}}\right|^{p}={ }^{r} \boldsymbol{T} \cdot\left|\boldsymbol{\Lambda}_{\hat{\boldsymbol{A}}_{r}}\right|^{p} \cdot{ }^{r} \boldsymbol{T}^{-1}=\left.\left.{ }^{r} T_{i q}\right|^{r} \lambda_{q}\right|^{p r} T_{q m}^{-1}
$$

where $\boldsymbol{\Lambda}_{\hat{A}}$ is the diagonal tensor of the eigenvalues ${ }^{r} \lambda_{q}$ of $\hat{A}_{r}$ and ${ }^{r} T_{i q}$ is the $i$ component of the eigenvector associated with the $q$ th eigenvalue. Notice that this eigenproblem has only real solution due to the symmetric form of $\hat{\boldsymbol{A}}_{r}$ as it can be seen in Eq. (18). Therefore, the calculation of $|\hat{\boldsymbol{A}}|_{p}$ is performed by Eq. (22) and its eigenproblem is solved as

$$
|\hat{\boldsymbol{A}}|_{p}=\boldsymbol{Y} \cdot \boldsymbol{\Lambda} \cdot \boldsymbol{Y}^{-1}
$$

where $\Lambda=\left[\lambda_{c_{p}}\right]$ is the diagonal tensor of the eigenvalues of $|\hat{\boldsymbol{A}}|_{p}$ and $\boldsymbol{Y}=\left[Y_{i q}\right]$ are the eigenvectors associated to them. The upwinding tensor will be defined in the basis of these eigenvectors. Firstly, it is necessary to describe the generalized diffusion tensor in the eigenvector system of the matrix-valued $p$-norm of the generalized convective tensor (the $\boldsymbol{Y}$ system). This tensor is called $\overline{\boldsymbol{K}}$ and it is defined as

$$
\overline{\boldsymbol{K}}=\left[\overline{\boldsymbol{K}}_{j n}\right]=\overline{\boldsymbol{K}}_{j i m n}=Y_{i q}^{-1} \hat{K}_{j q l n} Y_{l m}
$$

with $l=1, \ldots, n_{\mathrm{dim}}+1$.

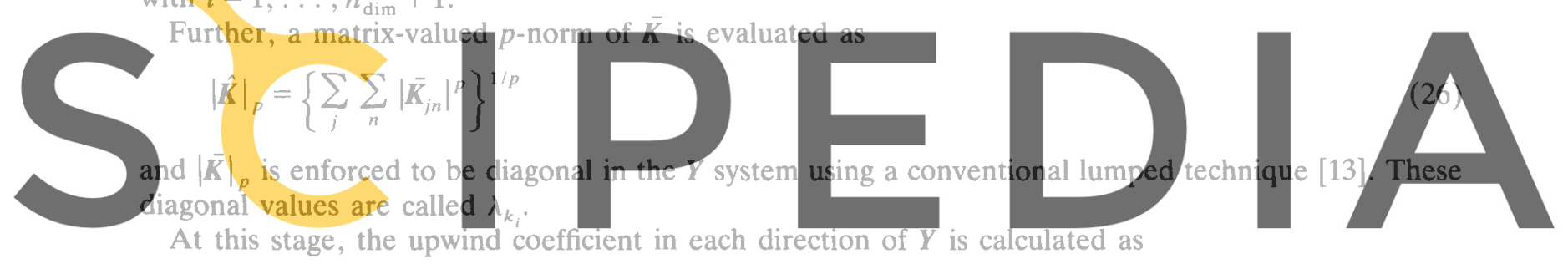

Register for free at https $\tau_{i}=$ / $/$ www.scipedia.com to download the version without the watermark

$$
\begin{array}{ll}
\text { - } \tau_{i}=\frac{\zeta\left(\alpha_{i}\right)}{\lambda_{c_{i}}} & \text { if } \lambda_{c_{i}} \neq 0 \text { and } \lambda_{k_{i}} \neq 0 \\
\text { - } \tau_{i}=\frac{1 .}{\lambda_{c_{i}}} & \text { if } \lambda_{c_{i}} \neq 0 \text { and } \lambda_{k_{i}}=0
\end{array}
$$

where $\zeta$ is the nondimensional numerical diffusivity optimal function defined by [13]

$$
\zeta\left(\alpha_{i}\right)=\operatorname{coth}\left(\alpha_{i}\right)-\alpha_{i}^{-1}
$$

and $\alpha_{i}$ is the elemental Péclet number

$$
\alpha_{i}=\frac{\lambda_{c_{i}}}{\lambda_{k_{i}}}
$$

The 'upwinding tensor' in system $Y$ is written as

$$
\tau_{Y}=\tau_{Y_{i j}}=\delta_{i k} \tau_{k} \delta_{k j}
$$

where $\delta$ is the Kronccker delta. Finally, the upwinding tensor used in Eqs. (11) and (12) is obtained transforming back this last expression as

$$
\boldsymbol{\tau}=\boldsymbol{Y} \cdot \boldsymbol{\tau}_{Y} \cdot \boldsymbol{Y}^{-1}
$$


It should be noted that this methodology obviously satisfies the three designs conditions proposed by Hughes et al. [5]: (i) it reduces correctly to the optimal one-dimensional system case; (ii) it is equivalent to SUPG for a scalar, multidimensional advection-diffusion equation; and (iii) it reduces to SUPG on each uncoupled component of multidimensional simultaneously diagonalizable advection-diffusion system (this implies that ${ }^{r} \boldsymbol{T}$ of Eq. (23) are the same for all $r$ ).

In particular, in the present work $p=1$ is adopted in the computation of the $p$-norm.

\section{Finite element formulation}

In the framework of the finite element method [13] the continuous field of unknowns $U$ are locally approximated by polinomial functions in the standard manner as

$$
U \simeq U_{h}=\Phi_{h} \hat{U}
$$

where $U_{h}$ is the approximation of the continuous unknown vector $U, \hat{U}$ is the nodal unknown vector and $\Phi_{h}$ are the typical shape functions for standard finite elements [13].

The spatial domain is discretized by a collection of $n_{\mathrm{cl}}$ elements disjointed such that the union of them is the original domain. Therefore, the continuous variational form of the problem described in Eq. (13) can be written in a semidiscrete form as [5]

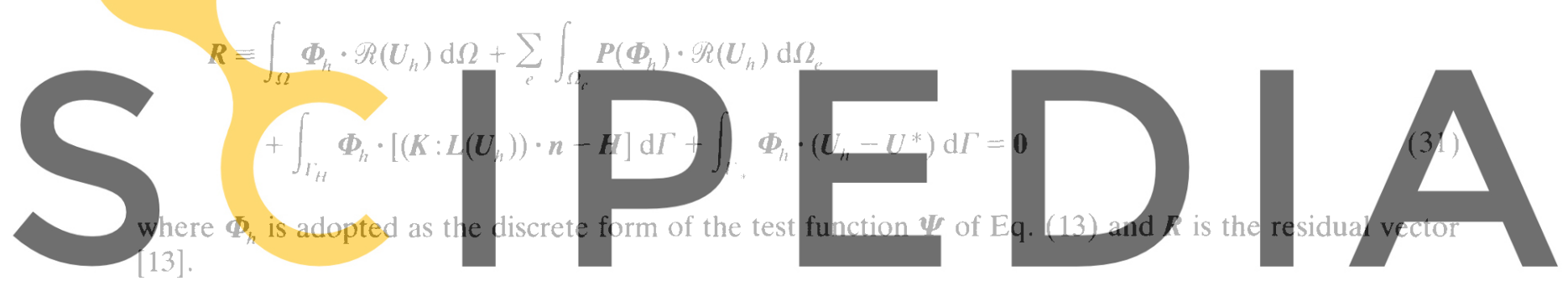

The temporal discretization of Eq. (31) has been done using the well-known Euler backward scheme Registerlffor free at https//www.scipedia.com to download the version without the watermark

\section{Solution strategy}

When the residual is differentiable, a Newton-type incremental-iterative formulation for solving the nonlinear semidiscrete system (31) can be attempted. This means that [13]

$$
\begin{aligned}
& { }^{t+\Delta t} \boldsymbol{J}^{j-1} \boldsymbol{\Delta} \boldsymbol{U}^{j}={ }^{t+\Delta t} \boldsymbol{R}^{\prime-1}, \\
& { }^{t+\Delta t} \boldsymbol{U}^{j}={ }^{t+\Delta t} \boldsymbol{U}^{j-1}+\boldsymbol{\Delta} \boldsymbol{U}^{j} \quad j=1, \ldots, n_{\mathrm{iter}} ; \\
& { }^{1+}{ }^{+} \boldsymbol{U}^{\prime}={ }^{\prime} \boldsymbol{U},
\end{aligned}
$$

where the iteration index $j$ denotes the $j$ th approximation to the solution in $t+\Delta t$ (the solution at time $t$ is assumed to be known), and $\boldsymbol{I}$ is the tangent Jacobian matrix $\boldsymbol{J}=-\partial \boldsymbol{R} / \partial \boldsymbol{U}$.

The convergence criterion is written in terms of the norm of the residual vector in the following form

$$
\frac{\|\boldsymbol{R}\|_{L_{2}}}{\left\|\boldsymbol{F}^{*}\right\|_{L_{2}}} \leqslant \varepsilon_{r}
$$

where $\varepsilon_{r}$ is the admissible tolerance (taken as $10^{-11}$ in this work), $F^{*}$ is a reference vector (the residual in the prescribed degrees of freedom or the body force vector if it exists) and $\|\cdot\|_{L_{2}}$ is the standard $L_{2}$ vector norm [13]. 


\section{Numerical examples}

\subsection{Driven cavity flow problem}

This example is a classical test used by several authors in order to check the quality of the methodology employed. In the present analysis, two different boundary conditions at the top corners are considered (see Fig. 2). The velocity is fixed in the walls and the pressure is taken equal to zero in the middle of the bottom. In the domain, the initial value of the unknowns are adopted equal to zero for all the cases presented. The geometry of the problem, the boundary conditions, the characteristic lengths and the meshes used in this work are shown in Figs. 2 and 3.

The stationary problem is considered for different Reynolds' numbers. The numerical results are compared with those obtained by

(a) Ghia et al. [14], no upwind F.D.M., $128 \times 128$ elements $(\mathrm{Re}=1000), 256 \times 256$ elements $(\operatorname{Re}=5000,10000)$.

(b) Nallasamy and Prasad [15], upwind F.D.M., $50 \times 50$ elements $(\operatorname{Re}=5000,10000)$

(c) Fortin and Thomasset [16], F.E.M., $12 \times 12$ elements $(\mathrm{Re}=1000)$.

(d) Bercovier and Engelman [17], no upwind F.E.M. with penalization, $12 \times 12$ elements $(\mathrm{Re}=$ 1000).

(e) Kondo et al. [18], third-order upwind F.E.M., $40 \times 40$ elements $(\operatorname{Re}=1000), 44 \times 44$ elements $(\operatorname{Re}=10000)$.

(f) Tanahashi et al. [19], GSMAC F.E.M.
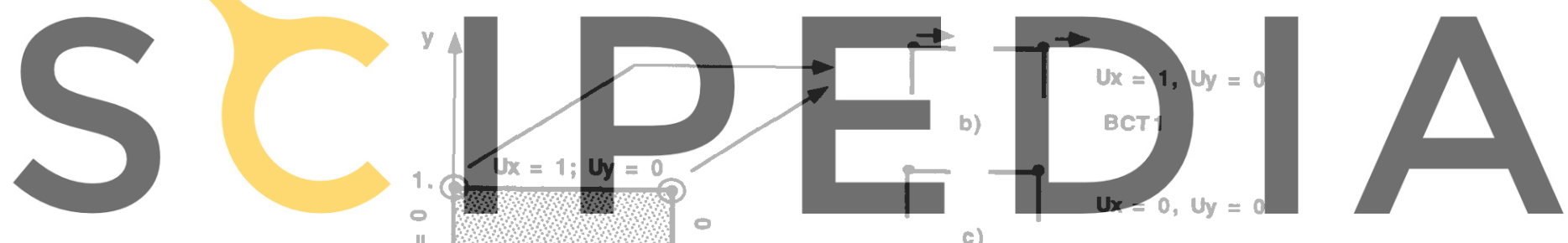

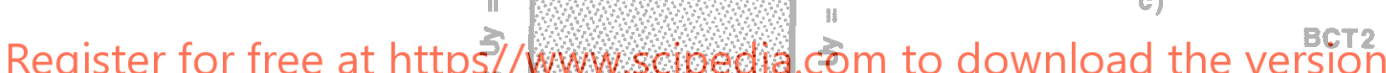

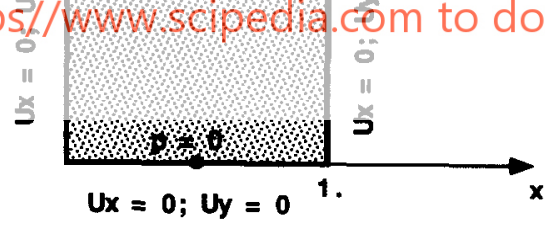

a)

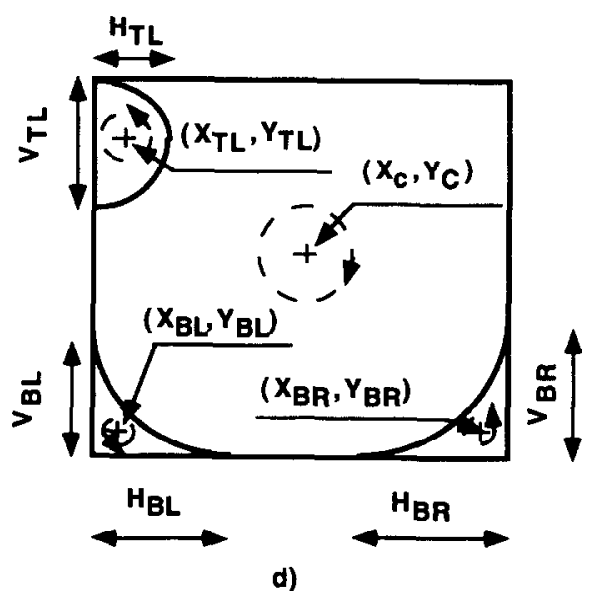

Fig. 2. Driven cavity flow. (a) Geometry; (b) boundary condition type 1 (BCT1); (c) boundary condition type 2 (BCT2); (d) characteristic lengths. 


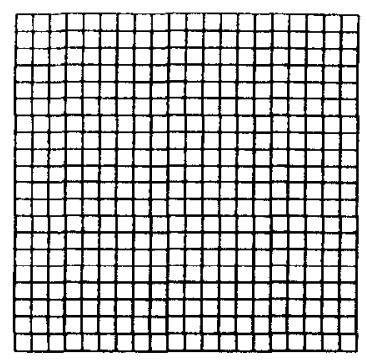

a)

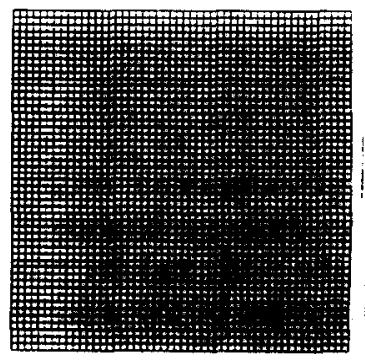

b)

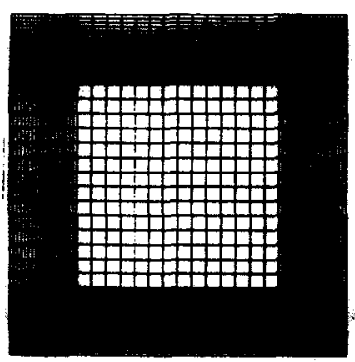

c)

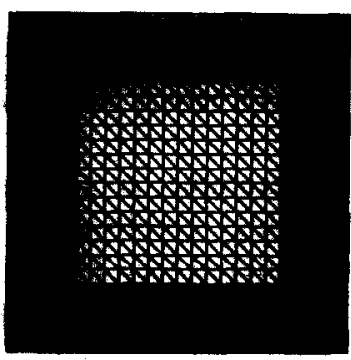

d)

Fig. 3. Driven cavity flow-Finite element meshes: (a) uniform, 400 four-noded elements; (b) uniform, 2500 four-noded elements; (c) nonuniform, 2500 four-noded elements; (d) nonuniform. 5000 three-noded elements.

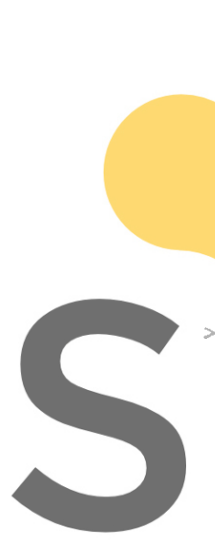

Register for f $\begin{array}{ccccccccc}-0.6 & -0.4 & -0.2 & 0 & 0.2 & 0.4 & 0.6 & 0.8\end{array}$

Ux

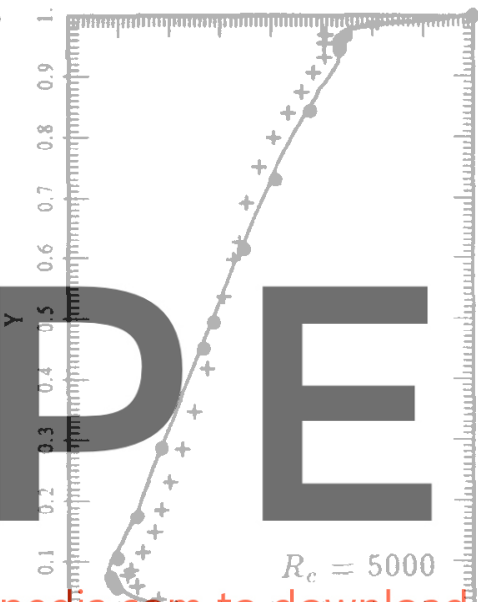

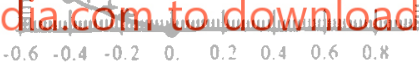

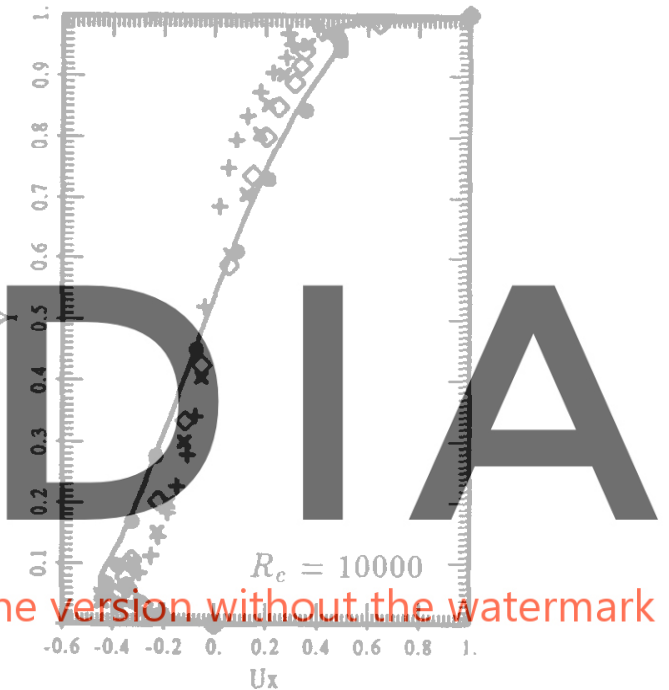

Ux

Fig. 4. Horizontal velocity profile for cavity flow at different Reynolds' numbers along the line $x=0.5$ with BCT2: $\bullet$, Ghia et al. [14]; $\times$, Nallasamy [15]: $\square$. Fortin and Thomasset [16]: $\bigcirc$. Bercovier and Engelman [17]; $\diamond$, Kondo et al. [18]; +, Tanahashi et al. [19]. Present work: _- - mesh (a); - - mesh (b); — mesh (c); _ _. - mesh (d).

Table 1

$\mathrm{Re}=1000-$ Steady-state analysis

\begin{tabular}{llll}
\hline & Tanahashi et al. [19] & Ghia et al. [14] & Present work \\
\hline$x_{\mathrm{r}}$ & 0.5335 & 0.5313 & 0.5409 \\
$y_{c}$ & 0.5653 & 0.5625 & 0.5855 \\
$x_{B R}$ & 0.8672 & 0.8594 & 0.8684 \\
$y_{B R}$ & 0.1119 & 0.1094 & 0.1072 \\
$x_{B L}$ & 0.0822 & 0.0859 & 0.0760 \\
$y_{B L}$ & 0.0731 & 0.0781 & 0.0754 \\
$H_{B R}$ & 0.3091 & 0.3034 & 0.3099 \\
$V_{B R}$ & 0.3410 & 0.3536 & 0.3710 \\
$H_{R L}$ & 0.2045 & 0.2188 & 0.2076 \\
$V_{B L}$ & 0.1523 & 0.1680 & 0.1826 \\
\hline
\end{tabular}



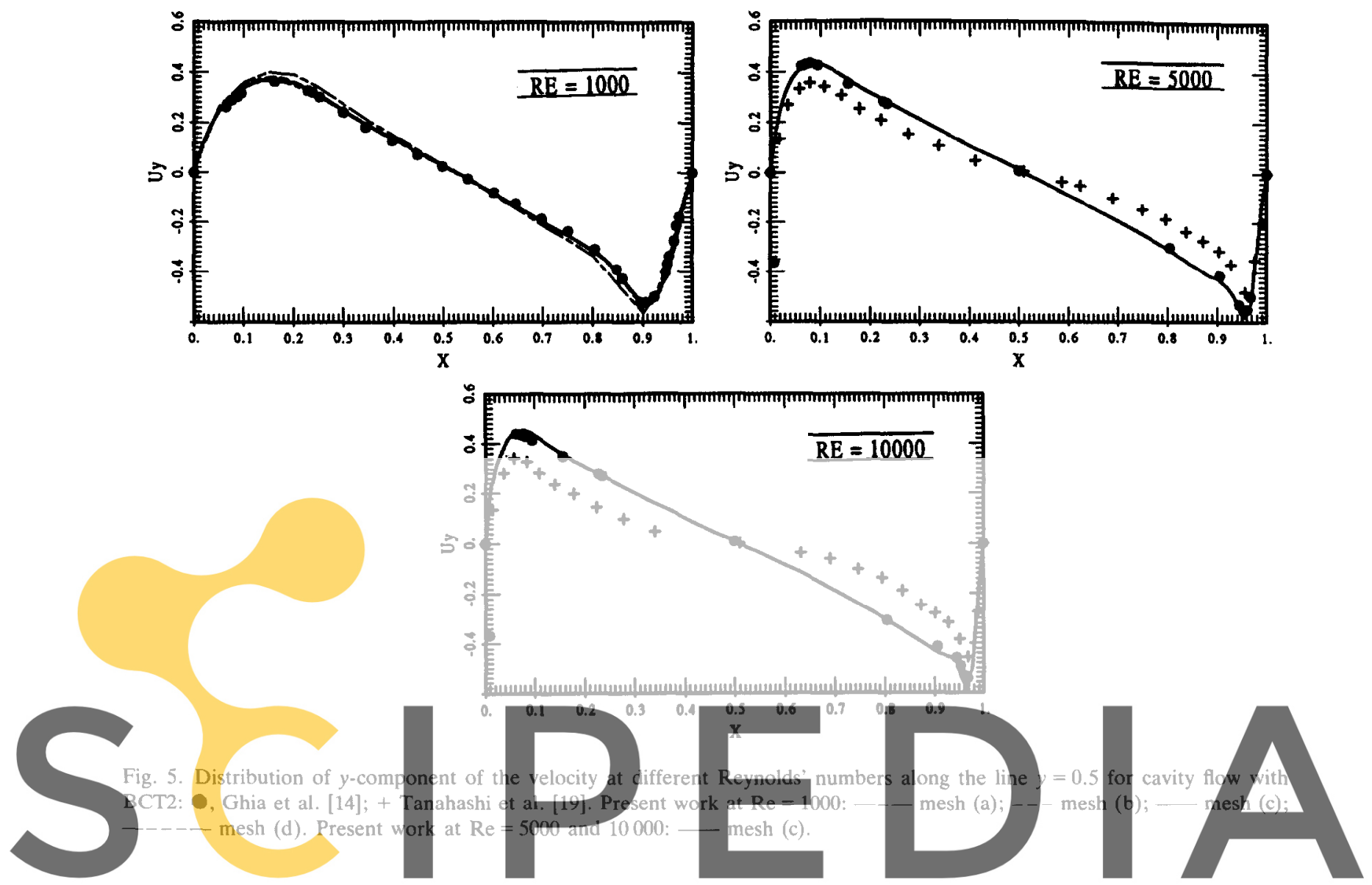

Fig. 4 shows the horizontal velocity profile along the line $x=0.5$ for $\operatorname{Re}=1000,5000$ and 10000 and

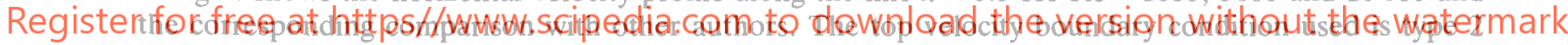
(see Fig. 2). In addition, the vertical velocity profile along the line $y=0.5$ is plotted in Fig. 5. The results obtained in the present work using different meshes are in very good agreement with Ghia et al. [14].

The characteristic lengths obtained with the present formulation are compared with the results of other authors and are shown in Tables $1-3$ for $R e=1000, R e=5000$ and $R e=10000$, respectively.

Table 2

$\operatorname{Re}=5000$-Steady-state analysis

\begin{tabular}{llll}
\hline & Tanahashi et al. [19] & Ghia et al. [14] & Present work \\
\hline$x_{c}$ & 0.5120 & 0.5117 & 0.5029 \\
$y_{C}$ & 0.5337 & 0.5352 & 0.5420 \\
$x_{B R}$ & 0.8134 & 0.8086 & 0.8012 \\
$y_{B R}$ & 0.0753 & 0.0742 & 0.0638 \\
$x_{B L}$ & 0.0750 & 0.0703 & 0.0754 \\
$y_{H L}$ & 0.1318 & 0.1367 & 0.1345 \\
$x_{T L}$ & 0.0658 & 0.0625 & 0.0585 \\
$y_{T L}$ & 0.9045 & 0.9102 & 0.9130 \\
$H_{B R}$ & 0.3496 & 0.3565 & 0.3623 \\
$V_{B R}$ & 0.4350 & 0.4180 & 0.4145 \\
$H_{B L}$ & 0.3159 & 0.3184 & 0.2923 \\
$V_{B L}$ & 0.2693 & 0.2643 & 0.2840 \\
$H_{T L}$ & 0.1208 & 0.1211 & 0.1101 \\
$V_{T L}$ & 0.2555 & 0.2693 & 0.2923 \\
\hline
\end{tabular}


Table 3

$\operatorname{Re}=10000-$ Steady-state analysis

\begin{tabular}{|c|c|c|c|}
\hline & Tanahashi et al. [19] & Ghia et al. [14] & Present work \\
\hline$x$ & 0.5125 & 0.5117 & 0.5000 \\
\hline$y_{\text {c }}$ & 0.5274 & 0.5333 & 0.5420 \\
\hline$x_{B R}$ & 0.7944 & 0.7656 & 0.7573 \\
\hline$y_{B R}$ & 0.0640 & 0.0586 & 0.0551 \\
\hline$x_{B t}$ & 0.0790 & 0.0586 & 0.0676 \\
\hline$y_{R t}$ & 0.1400 & 0.1641 & 0.1536 \\
\hline$x_{T L}$ & 0.0758 & 0.0703 & 0.0676 \\
\hline$y_{T I}$ & 0.9120 & 0.9141 & 0.9130 \\
\hline$H_{B R}$ & 0.3773 & 0.3906 & 0.3655 \\
\hline$V_{B R}^{B R}$ & 0.4529 & 0.4492 & 0.4522 \\
\hline$H_{R L}$ & 0.3515 & 0.3438 & 0.3216 \\
\hline$V_{H i}$ & 0.2834 & 0.2891 & 0.2899 \\
\hline$H_{r l}$ & 0.1683 & 0.1589 & 0.1491 \\
\hline$V^{\prime}$ & 0.3463 & 0.3203 & 0.3333 \\
\hline
\end{tabular}
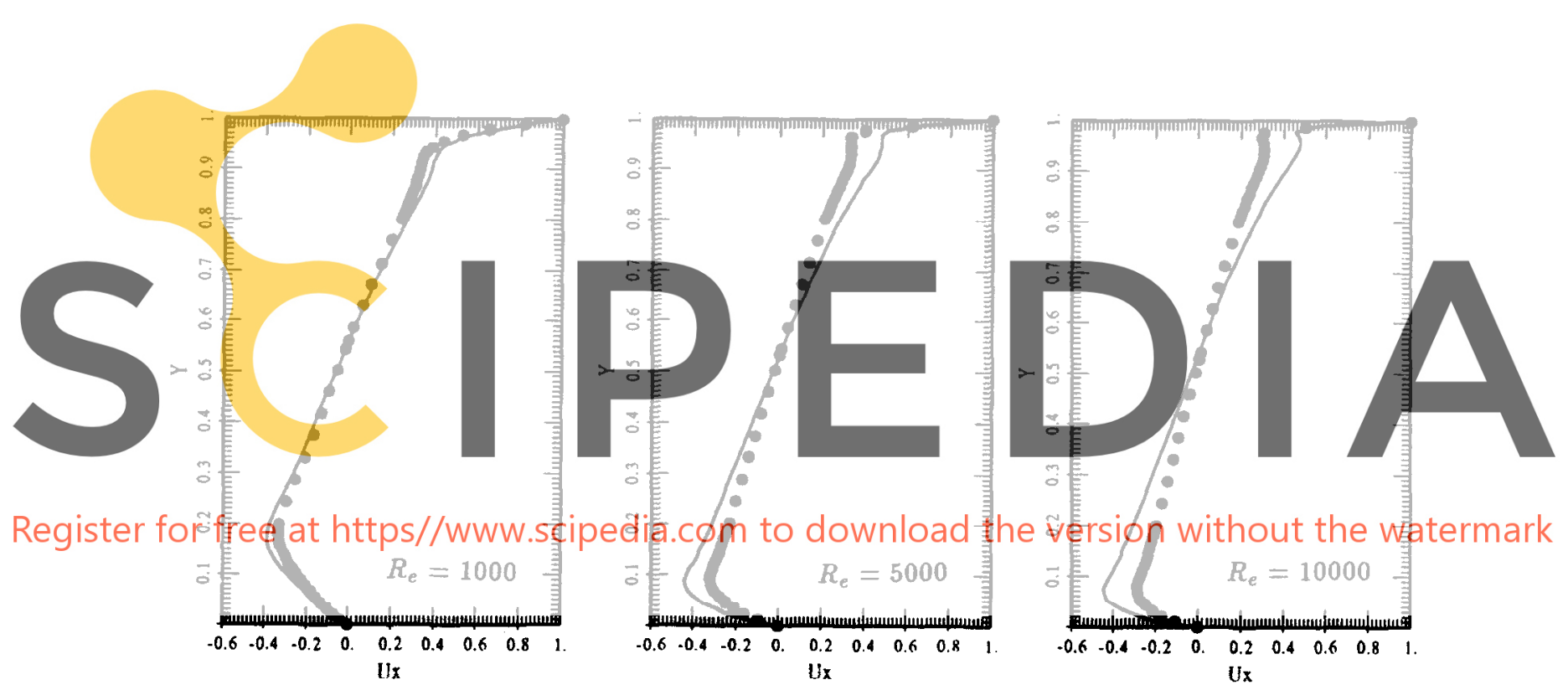

a) $x$-component of the velocity along the line $x=0.5$.
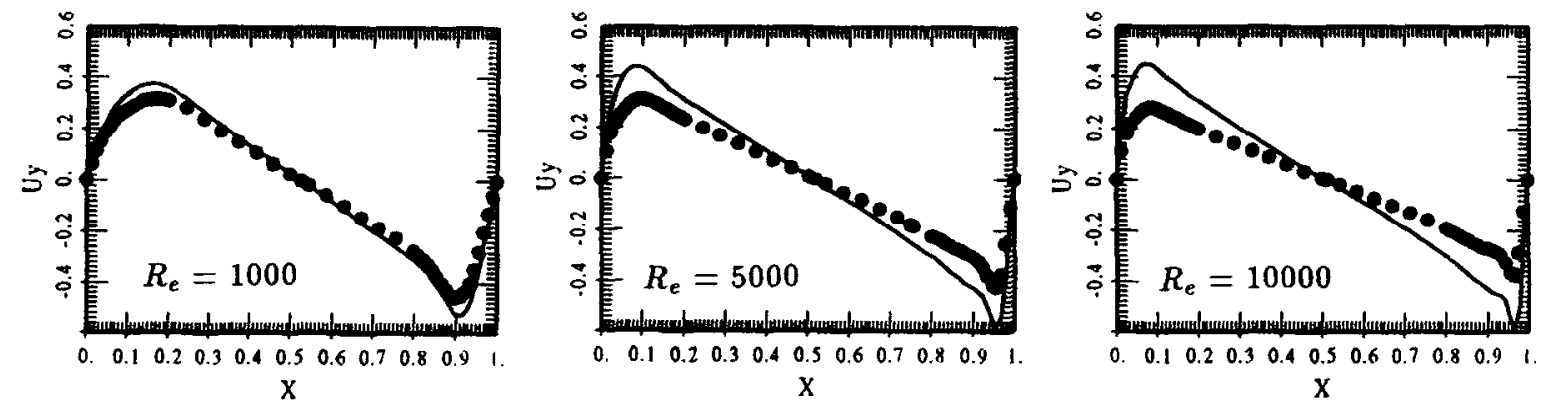

b) y-component of the velocity along the line $y=0.5$.

Fig. 6. Driven cavity flow problem-BCT1 (๑) in comparison with BCT2 ( - ) at different Reynolds' numbers with the formulation presented in this work using mesh (c). 
M.A. Cruchaga, E. Oñate / Comput. Methods Appl. Mech. Engrg. 143 (1997) 49-67
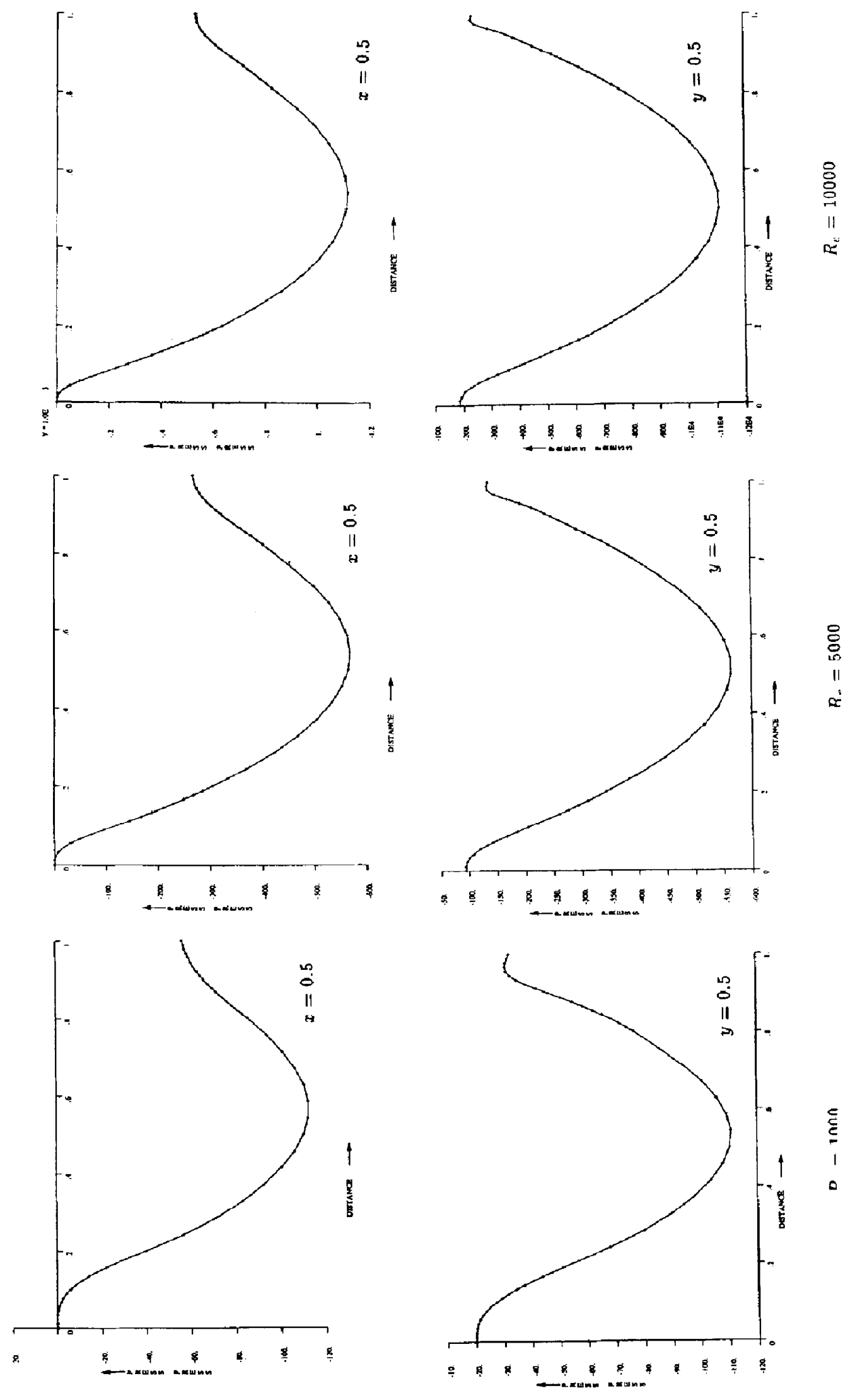

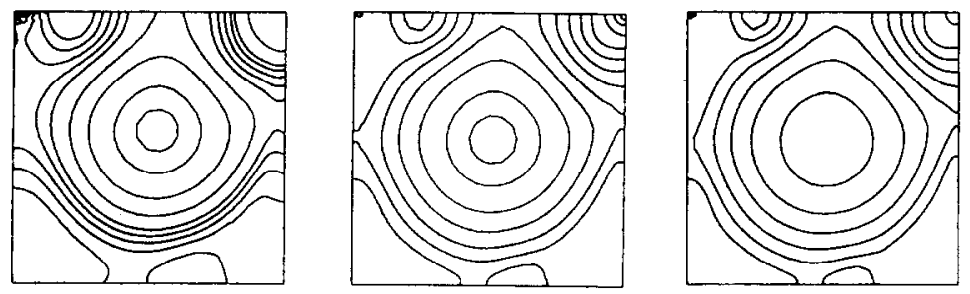

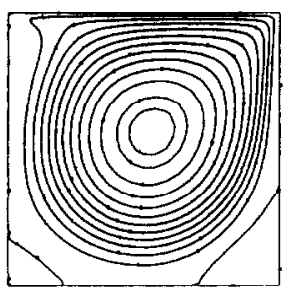

$R_{\mathrm{r}}=1000$

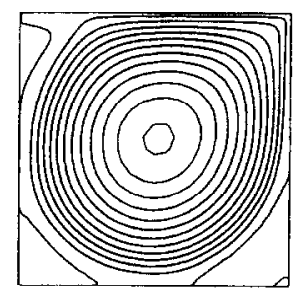

$R_{e}=5000$

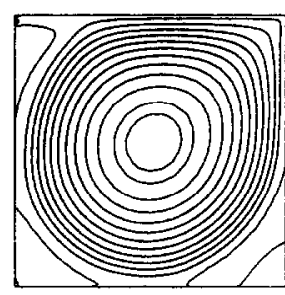

$R_{e}=10000$

Fig. 8. Cavity flow problem-Pressure contours and streamlines.

The effect of the top boundary condition at different Reynolds' numbers is presented in Fig. 6 .

The results obtained for $\mathrm{Re}=1000,5000$ and 10000 using a scalar upwinding GLS-type technique $[11,12]$ are similar to those computed using the present methodology with the same numerical strategy.

The pressure profile along the lines $x=0.5$ and $y=0.5$ are plotted for different Reynolds' numbers in Fig. 7. It is seen that there are not oscillations in these profiles. Fig. 8 shows the streamline and pressure contours at different Reynolds' numbers. Once more, the numerical response does not present significant oscillations.

In addition, the values for the streamline function $(\psi)$ are presented in Tables $4-6$ for the Reynolds'

Table 4

$\mathrm{Re}=1000-$ Steady-state analysis. Streamvalues

\begin{tabular}{lcc}
\hline & Ghia et al. [14] & Present work \\
\hline$\psi_{c}$ & -0.117929 & -0.118 \\
$\psi_{B R}$ & $1.7510210^{3}$ & $1.7510^{*}$ \\
$\psi_{B L}$ & $2.3112910^{-4}$ & $2.2510^{\circ}$ \\
\hline
\end{tabular}

Table 5

$\operatorname{Re}=5000-$ Steady-state analysis. Streamvalues

\begin{tabular}{lcc}
\hline & Ghia et al. [14] & Present work \\
\hline$\psi_{c}$ & -0.118966 & -0.1215 \\
$\psi_{B R}$ & $3.0835810^{-3}$ & $3.2710^{3}$ \\
$\psi_{B L}$ & $1.3611910^{3}$ & $1.310^{-3}$ \\
$\psi_{T l}$ & $1.4564110^{-3}$ & $1.2910^{3}$ \\
$\psi_{B R(2)}$ & $-1.4322610^{\circ}$ & $-1.610^{-1}$ \\
\hline
\end{tabular}

Table 6

$\operatorname{Re}=10000$-Steady-state analysis. Streamvalues

\begin{tabular}{lcc}
\hline & Ghia et al. [14] & Present work \\
\hline$\psi_{i}$ & -0.119731 & -0.1197 \\
$\psi_{B R}$ & $3.4183110^{3}$ & $3.7910^{-3}$ \\
$\psi_{B L}$ & $1.5182910^{-3}$ & $1.3810^{3}$ \\
$\psi_{T i}$ & $2.4210310^{-3}$ & $2.310^{3}$ \\
$\psi_{B R}$ & $-1.3132110^{-4}$ & $-2.310^{*}$ \\
\hline
\end{tabular}


Table 7

Vorticity values

\begin{tabular}{rll}
\hline Re & Ghia et al. [14] & Present work \\
\hline 1000 & 2.04968 & 2.07 \\
5000 & 1.86016 & 1.9 \\
10000 & 1.88082 & 1.8 \\
\hline
\end{tabular}

numbers 1000,5000 and 10000 , respectively. The vorticity values in the main vortex at different Reynolds' numbers are presented in Table 7.

\subsection{Backward-facing step flow problem}

The backward-facing step flow problem is a very interesting test in order to compare the numerical results with the experimental ones obtained by Armaly et al. [20]. In the present analysis, the effect of the gravity action (in the vertical direction) is taking into account in order to reproduce the experimental test. The geometry and the characteristic lengths defined in this problem [20] are plotted in Fig. 9. A regular (structured) mesh composed of nearly 9000 four-noded bilinear isoparametric elements have been used in the computations. The velocity is prescribed to zero in the channel walls and a parabolic profile with maximum velocity $V_{\max }$ is considered at the inlet face. The velocity field is not restricted at the exit. The pressure is zero at the top corner in the outlet face. The air properties are taken as $\mu=0.000018$ for the dynamic viscosity and $\rho=1.2$ for the density (all in consistent units). The Reynolds' numbers are computed from the maximum inlet velocities $\left(V_{\max }\right)$ and the hydraulic diameter of the inlet channel $(D)$ as $\operatorname{Re}=\left(2 V_{\max } D \rho\right) /(3 \mu)$ [21]. The streamline and the velocity contours are presented in Fig. 10. In Table 8 the numerical results obtained in the present work and the experimental ones [20] are presented. It is possible to achieve convergence for high Reynolds' numbers but the disagreement between the experimental and numerical results, in particular for $\operatorname{Re}=1600$, can be due to the laminar numerical model used. Other reasons for these differences is the effect of the three dimensionality in the experimental results [20]. On the other hand, the numerical results are similar to that obtained in [21] using a traditional SUPG method with penalization and applying a continuation technique: $10.3,10.8$ and 17.2 for $x_{i} / s, i=1,2,3$ at $\operatorname{Re}=800$; and $12.8,13.0$ and 22.1 for the same characteristic lengths at $\operatorname{Re}=1000$. Fig. 11 shows the pressure contours under the gravity action. It should be noted that vertical isopressure lines near the inlet and the outlet would be obtained if the gravity action is not considered [21]. Finally, the same analysis have been performed in $[11,12]$ using a scalar upwinding GLS technique leading to similar results in comparison with those obtained using the present formulation.

\subsection{Two-liquid interface problem}

This problem has also been analysed in [22] and [10]. Two liquids with the same dynamic viscosity and different densities equal to 1.0 and 2.0 , respectively, occupy a closed tank with dimensions $0.8 \times 0.6$

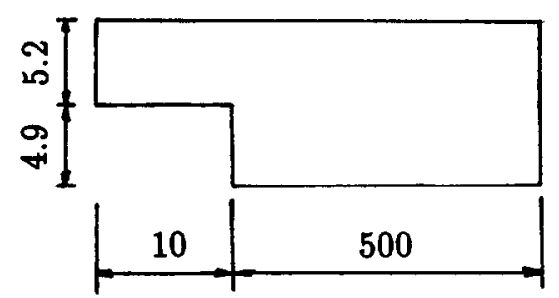

a)

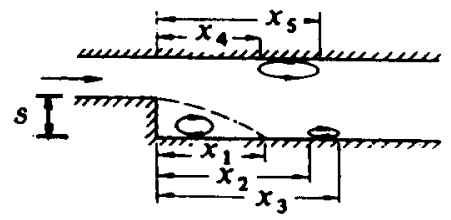

b)

Fig. 9. Backward-facing step flow. (a) Geometry (out of scale) in mm and (b) characteristic lengths. 

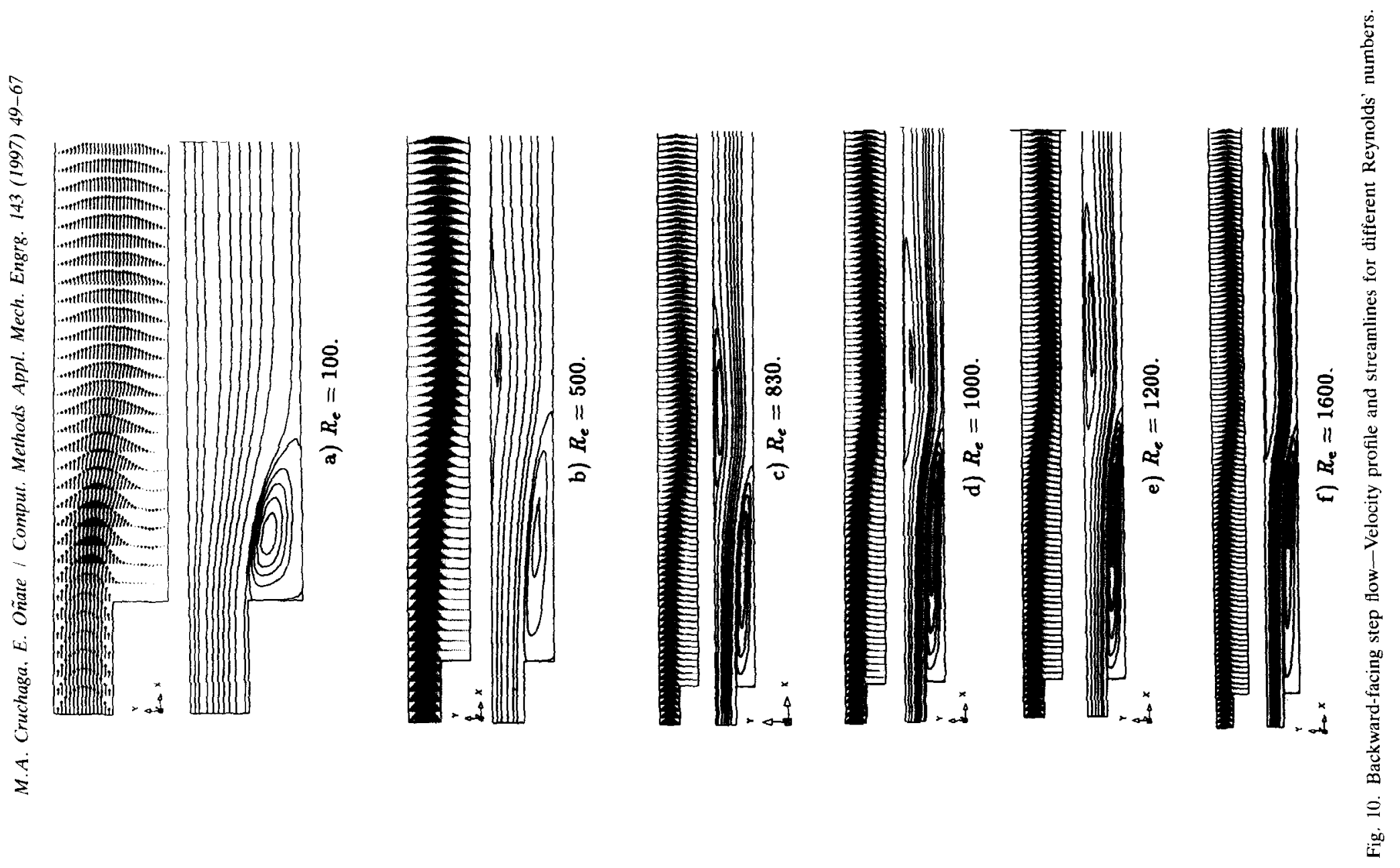
Table 8

Backward-facing step flow at different Reynolds' numbers

\begin{tabular}{lcccc}
\hline & $\mathrm{Re}$ & $x_{1} / s$ & $x_{4} / s$ & $x_{5} / s$ \\
\hline Present work & 100 & 3.0 & & \\
Armaly et al. [20] & 100 & 3.0 & & \\
Present work & 500 & 8.5 & 8.0 & 12.8 \\
Armaly et al. [20] & 500 & 10.0 & 8.0 & 13.5 \\
Present work & 830 & 11.5 & 10.5 & 19.4 \\
Armaly et al. [20] & 830 & 14.0 & 11.25 & 20.0 \\
Present work & 1000 & 12.93 & 11.22 & 22.79 \\
Armaly et al. [20] & 1000 & 16.25 & 13.5 & 21.8 \\
Present work & 1200 & 14.6 & 13.3 & 25.5 \\
Armaly et al. [20] & 1200 & 17.6 & 14.5 & 23.5 \\
Present work & 1600 & 17.0 & 14.6 & 34.0 \\
Armaly et al. [20] & 1600 & 13.9 & 9.5 & 22.0 \\
\hline
\end{tabular}

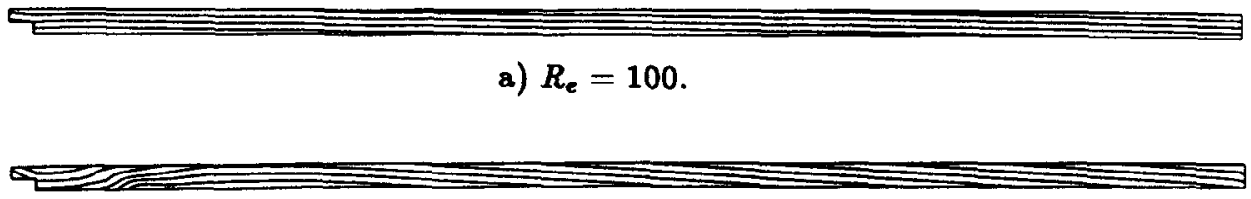

b) $R_{e}=500$.

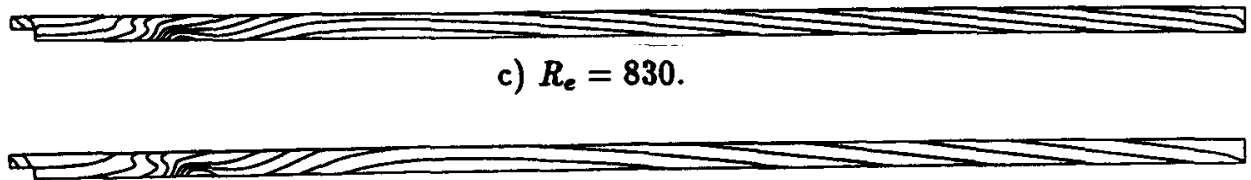

d) $R_{e}=1000$.

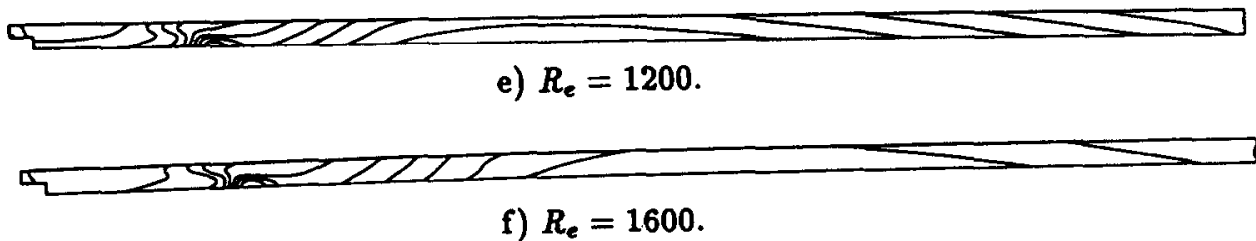

Fig. 11. Backward-facing step flow-Pressure contours.

(all in consistent units). The initial interface position is linear with a slope of 0.25 and average height of 0.3. The lighter liquid is on top of the heavier one and the gravity is 0.294 . The analysis performed is transient with a time step equal to 0.5. The geometry and the four-noded finite element mesh used are shown in Fig. 12. The normal velocity is prescribed equal to zero in all sides of the tank while the tangencial component is set to zero at the top and bottom sides (BCT1). The pressure is taken equal to zero at the top right corner. The interface position is obtained using the methodology developed in [23], consisting in following the interface by means of an arbitrary lagrangian mesh using the total velocity of the fluid particles belonging to it. The vertical location of the interface along the sides of the tank are plotted in Fig. 13. A very good agreement with the results obtained by Tezduyar et al. [22] can be observed. The pressure at different time steps are shown in Fig. 14. Once more, these results are very similar to those obtained in [22]. The results obtained prescribing only the normal velocity to zero on the top and bottom sides of the tank (BCT2) are plotted in Fig. 15 and they are compared with the results computed using the present methodology with BCT1. Fig. 16 shows the results obtained with the methodology presented in this work and those computed with a scalar upwinding GLS-type techniques 


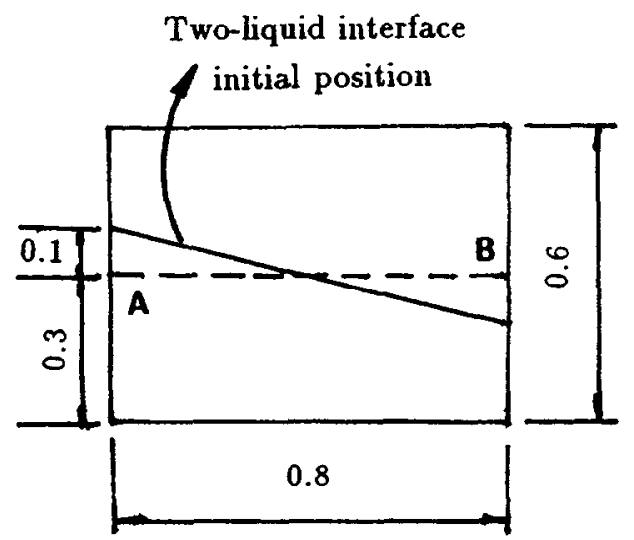

a)

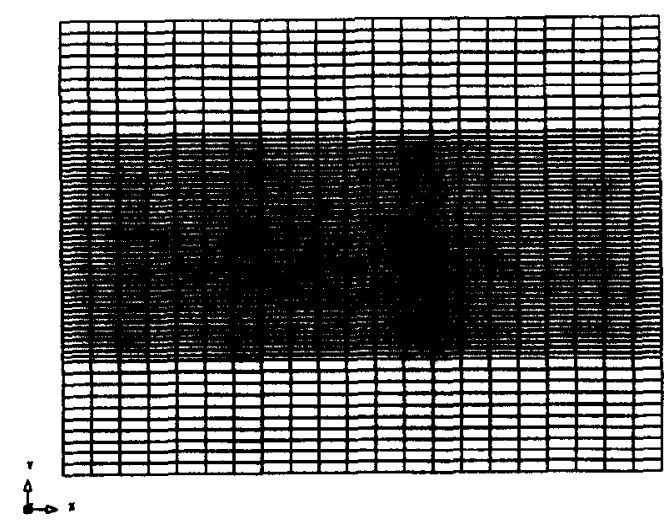

b)

Fig. 12. Two-liquid interface-Geometry (a) and four-noded finite element mesh (b)

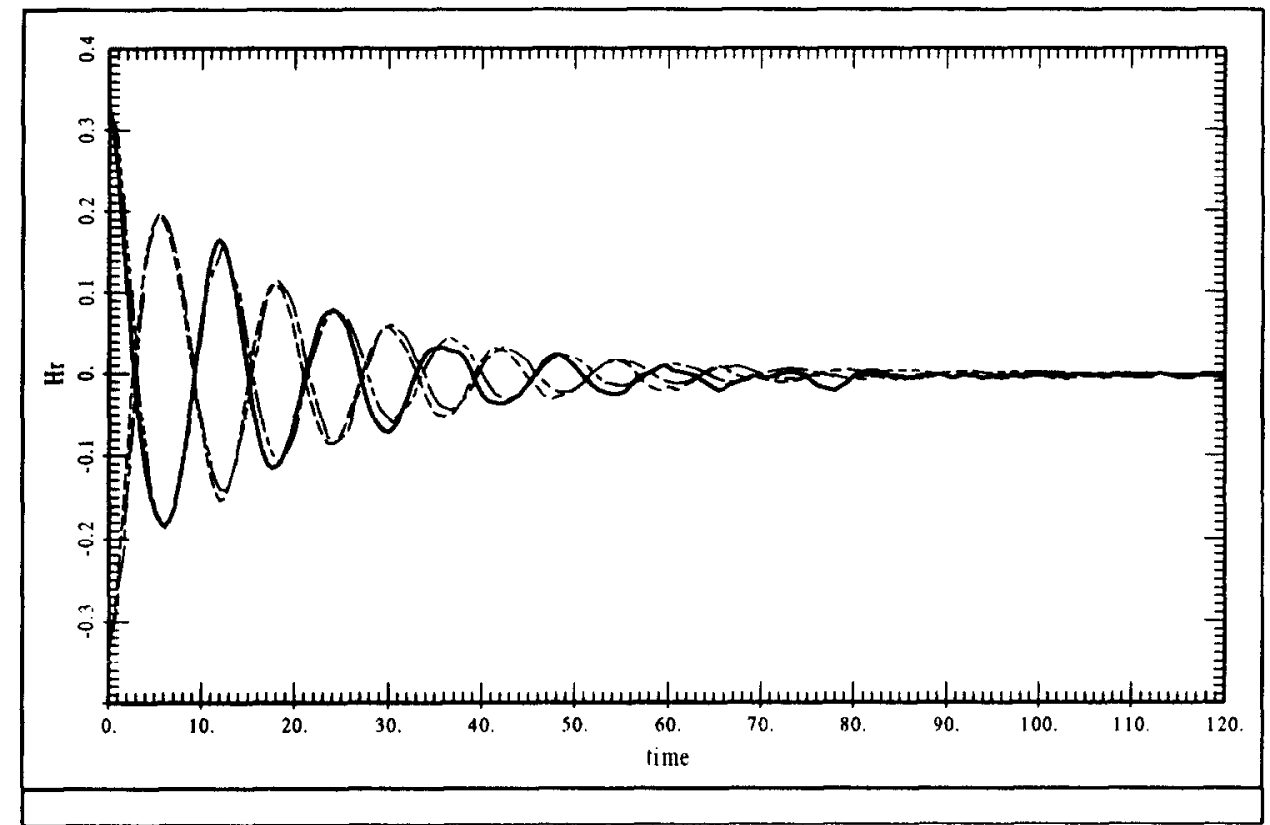

Fig. 13. Two-liquid interface-Time history of the interface positions in side $\mathrm{A}$ and side $\mathrm{B}$ of the tank. Side A: Tezduyar et al. [22]; —, present work. Side B: - - . Tezduyar et al. [22], --- , present work. $\left(H_{r}=(\right.$ wave height -0.3$)$ $0.3)$.

$[11,12]$ (using in both cases BCT1 and the same numerical strategy). Note that in the second case there are difficulties to reach the incompressibility condition.

\section{Conclusions}

A finite element GLS type formulation for solving the incompressible Navier-Stokes flow equations has been developed. In this context, the state variables of the problem are the velocity and pressure. This new methodology is based on the generalized streamline operator including the definition of an upwinding tensor which does not require input tuning parameters. Another features of this technique 


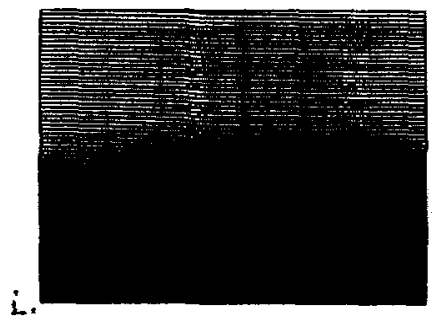

Step 18

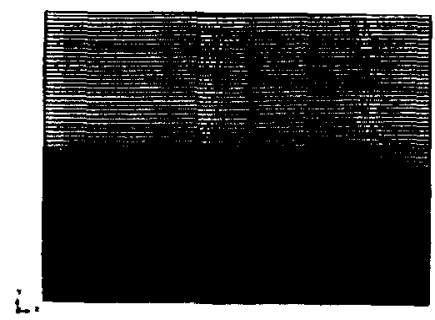

Step 30

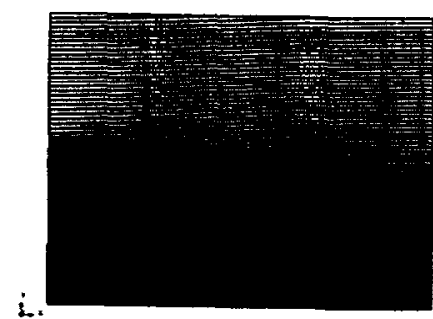

Step 24

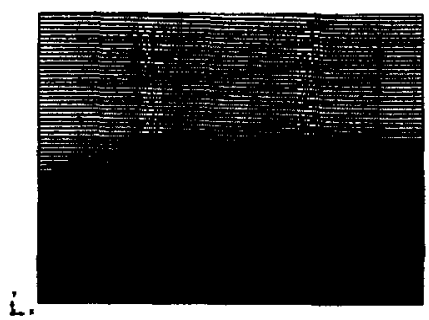

Step 36

Fig. 14. Two-liquid interface-Pressure contours.

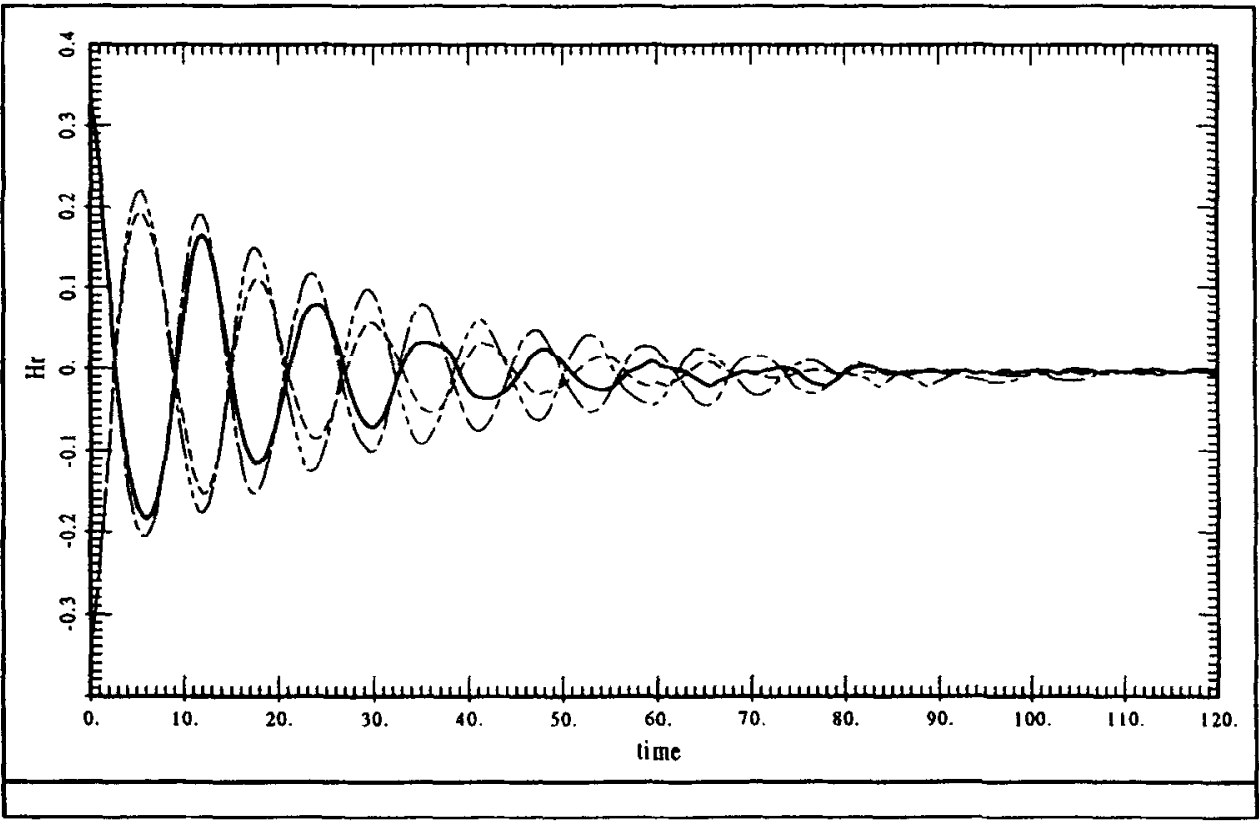

Fig. 15. Two-liquid interface-Time history of the interface positions in side A and side B of the $\operatorname{tank}$. Side A: - - BCT1; -- , BCT2. Side B: ---, BCT1; - - - BCT2. $\left(H_{r}=(\right.$ wave height -0.3$\left.) / 0.3\right)$.

are: it allows the use of equal order interpolation for velocity and pressure, no penalization methods are needed to satisfy the incompressibility condition and it reduces to the standard SUPG method for some particular cases related to the upwinding design conditions. Moreover, this formulation has been solved using a Newton-type incremental-iterative numerical scheme where the convergence criterion is written in terms of the residual vector. Finally, the numerical examples presented show a good agreement between the results obtained using the present formulation with some experimental measurements and other numerical results reported by different authors. 


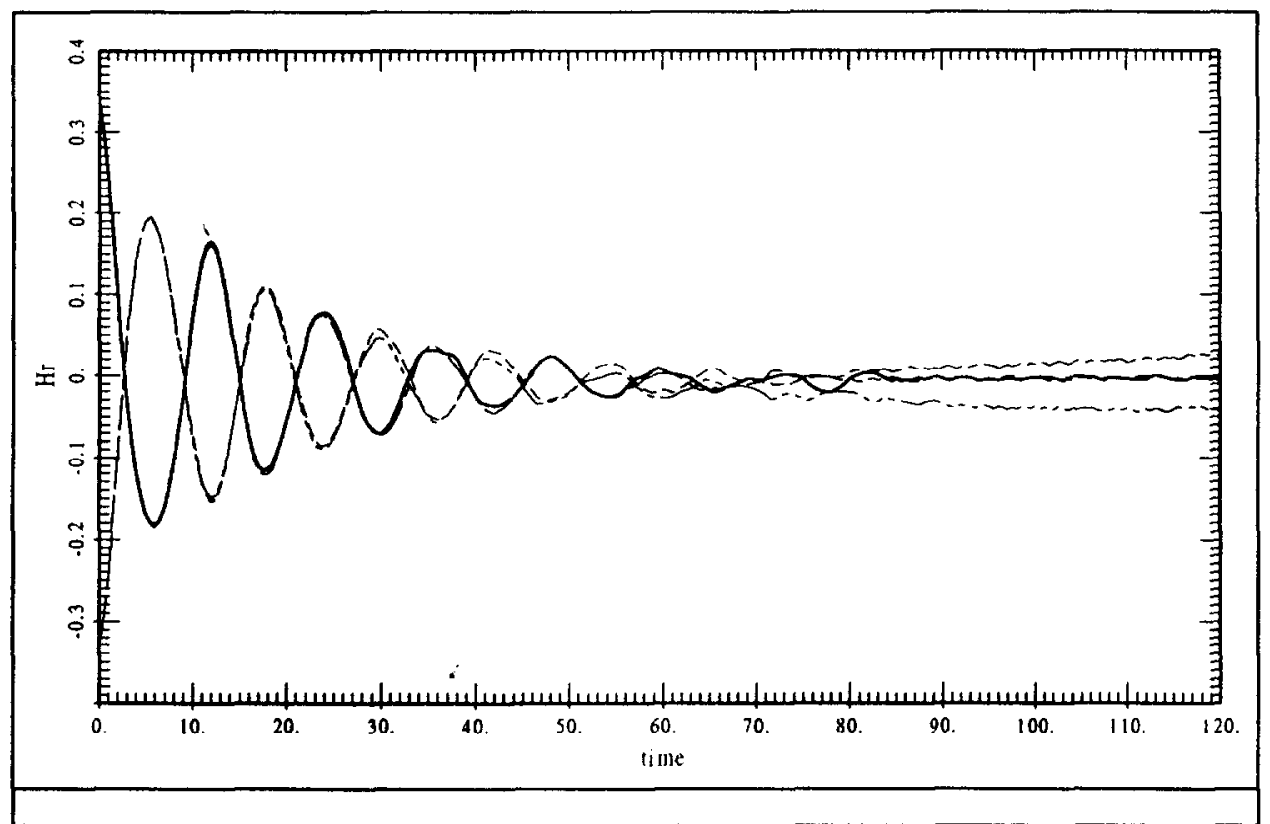

Fig. 16. Two-liquid interface-Time history of the interface positions in side A and side B of the tank. Side A: - Present work; - - Cruchaga and Oñate $[19,20]$. Side B: - - , Present work; - - - - Cruchaga and Oñate $[11,12] .\left(H_{r}=(\right.$ wave height -0.3$) / 0.3)$.

\section{Acknowledgment}

The first author thanks Francisco Armero for many useful discussions related with some specific topics of this work.

\section{References}

[1] T.J.R. Hughes, L.P. Franca and M. Balestra, A new finite element formulation for computational fluid dynamics: V. Circumventing the Babuska-Brezzi condition: a stable Petrov-Galerkin formulation of the stokes problem accommodating equal-order interpolations, Comput. Methods Appl. Mech. Engrg. 59 (1986) 85-99.

[2] T.J.R. Hughes, L.P. Franca and G.M. Hulbert, A new finite element formulation for computational fluid dynamics: VIIl. The Galerkin/least-squares method for advective-diffusive equations, Comput. Methods Appl. Mech. Engrg. 73 (1989) $173-189$.

[3] O.C. Zienkiewicz and J. Wu, Incompressibility without tears-How to avoid restrictions of mixed formulation, Int. J. Numer. Methods Engrg. 32 (1991) 1189-1203.

[4] L.P. Franca and T.J.R. Hughes, Convergence analyses of Galerkin least-squares methods for symmetric advective-diffusive forms of the Stokes and incompressible Navier-Stokes equations, Comput. Methods Appl. Mech. Engrg. 105 (1993) $285-298$.

[5] T.J.R. Hughes and M. Mallet, A new finite element formulation for computational fluid dynamics: III. The generalized streamline operator for multidimensional advective-diffusive systems, Comput. Methods Appl. Mech. Engrg. 58 (1986) 305-328.

[6] T.E. Tezduyar, M. Behr and J. Liou, A new strategy for finite element computations involving moving boundaries and interfaces-The deforming-spatial-domain/space-time procedure: I. The concept and the preliminary numerical tests, Comput. Methods Appl. Mcch. Engrg. 94 (1992) 339-351.

[7] P.A.B. De Sampaio, A Petrov-Galerkin formulation for the incompressible Navier-Stokes equations using equal order interpolation for velocity and pressure, Int. J. Numer. Methods Engrg. 31 (1991) 1135-1149.

[8] M. Storti, N. Nigro and S. Idelsohn, Stabilizing equal-order interpolations for mixed formulations of Navier-Stokes equations via SUPG method, Internal report.

[9] G. Hauke and T.J.R. Hughes, A unified approach to compressible and incompressible flows, Comput. Methods Appl. Mech. Engrg. 113 (1994) 389-395. 
[10] M.A. Cruchaga and E. Oñate, A finite element formulation for incompressible flow with moving surfaces using a generalized streamline operator, Proc. Ninth International Conference on Finite Elements in Fluids, 1995.

[11] M.A. Cruchaga and E. Oñate, A numerical solution strategy for the incompressible Navier-Stokes equations. Modeling and simulation, Proc. 1994 European Simulation Multiconference (1994) 699-703.

[12] M.A. Cruchaga and E. Oñate, Modelización por elementos finitos de las ecuaciones de Navier-Stokes para flujo incompresible mediante técnicas GLS, Mecánica computacional, ed. (AMCA, Santa Fe, Argentina) 14 (1994) $255-267$.

[13] O.C. Zienkiewicz and R.L. Taylor, The Finite Element Method, Fourth edition (McGraw-Hill Book Company).

[14] U. Ghia, K.N. Ghia and C.T. Shin, High-Re solutions for incompressible flow using the Navier-Stokes equation and multigrid method, J. Comput. Phys. 48 (1982) 387-411.

[15] M. Nallasamy and K.K. Prasad, On cavity flow at high Reynolds numbers, J. Fluid Mech. 79 (1977) $391-414$.

[16] M. Fortin and F. Thomasset, Mixed finite element methods for incompressible flow problems, J. Comput. Phys. 31 (1979) $113-145$.

[17] M. Bercovier and M. Engelman, A finite element for the numerical solution of viscous incompressible flows, J. Comput. Phys. 30 (1979) 181-201.

[18] N. Kondo, T. Tosaka and T. Nishimura, High Reynolds solutions of the Navier-Stokes equations using the third-order upwind finite element method, Proc. Computacional Methods in Flow Analysis (Okayama, 1988) 984-911.

[19] T. Tanaheshi, H. Okanaga and T. Saito, GSMAC Finite Element Method for unsteady incompressible Navier-Stokes equations at high Reynolds numbers, Int. J. Numer. Methods in Fluids 11 (1990) 479-499.

[20] B.F. Armaly, F. Durst, J.C.F. Pereira and B. Schönung, Experimental and theoretical investigation of backward-facing step flow. J. Fluid Mech. 127 (1983) 473-496.

[21] R. Codina, A Finite Element model for incompressible flow problems, Doctoral Thesis, Barcelona, June, 1992.

[22] T.E. Tezduyar, M. Behr and J. Liou, A new strategy for finite element computations involving moving boundaries and interfaces. The deforming-spatial-domain/space-time procedure: II. Computation of free-surface flows two-liquid flows, and flows with drifting cylinders. Comput. Methods Appl. Mech. Engrg. 94 (1992) 339-351.

[23] M. Cruchaga, E. Oñate and S. Idelsohn, On the pseudomaterial approach for the analysis of transient forming processes, Comput. Numer. Methods Engrg. 11 (1995) 137-148. 